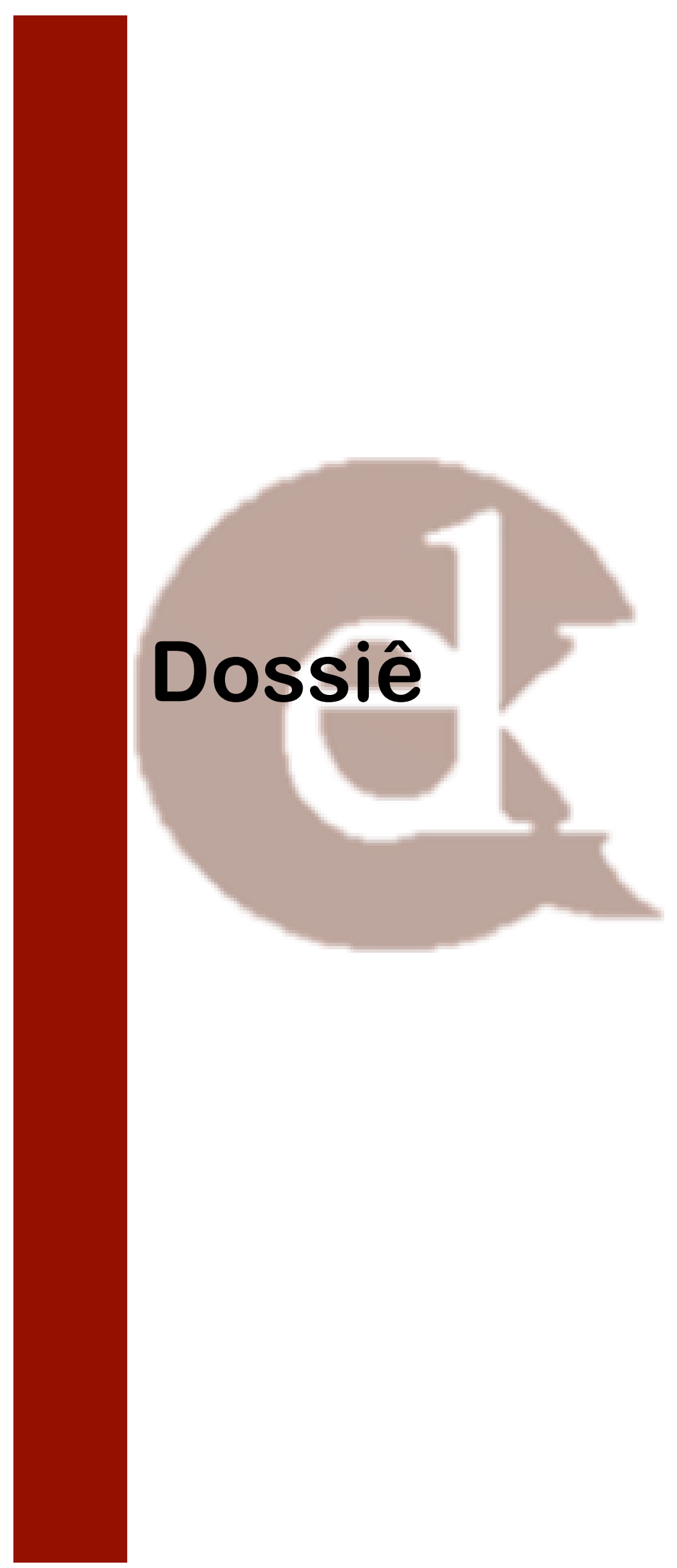




\title{
Martin Heideggers Festrede Gelassenheit: die Frage nach dem Wesen der Technik und dem Denken des Menschen
}

DOI: 10.12957/ek.2020.53266

\author{
Prof. Dr. Alfred Denker ${ }^{1}$ \\ Martin-Heidegger-Archiv, Meßkirch \\ Archivo-Heidegger, Universidad de Sevilla \\ alfred.denker@yahoo.com
}

\begin{abstract}
The main objective of this article is to elucidate, from the discourse Gelassenheit, which occurred in 1955, problems that pervade Martin Heidegger's late philosophy, especially with regard to the issue of technique and human thought. To problematize the technique implies establishing the distinction between meditative thinking and calculating thinking, in addition to calling into question the position of the human being in the totality proper to the entity. The technique would therefore be a way of claiming the individual and a transformation of his essence, as well as one of the faces of the consummation of nihilism present in the current era.
\end{abstract}

Keywords: Serenity. Technique. Heidegger. Historical Thought of Being.

\footnotetext{
${ }^{1}$ Agradecemos ao Professor Dr. Alfred Denker, pela gentileza em compor conosco o presente dossiê, remetendo este seu escrito inédito, de valor inestimável. Na edição do artigo, decidimos manter as citações conforme o material que nos foi originalmente enviado. No entanto, foi necessário padronizar alguns aspectos pré-textuais e formais, como a inserção do resumo e das palavras-chave, e a formatação geral do texto. As referências bibliográficas serão mantidas conforme o manuscrito original: nas notas de rodapé. [Nota das(os) editoras(es)].
} 
Für Heidegger war die Festrede „Gelassenheit“ zum 175. Geburtstag des Meßkircher Komponisten Conradin Kreutzer (1780-1849) am 30. Oktober 1955 in Meßkirch ein besonderes Ereignis. Seit seinen ganz frühen Vorträgen in Meßkirch als Theologie- und Philosophiestudent hatte er nicht mehr öffentlich in Meßkirch für seine Heimatleute gesprochen. ${ }^{2}$ Er hatte die Einladung von Bürgermeister Schühle nur unter der Bedingung, dass er das Thema freiwählen dürfte, angenommen. Heidegger wollte selbstverständlich keine übliche Gedenkrede halten. Ein wichtiger Teil der Gedenkfeier war die Aufführung von Kreutzers Musik, neben Lied und Chor, auch Oper und Kammermusik. So wurde Conradin Kreutzer als Künstler und Meister der Musik gefeiert. Wie fast immer fängt Heidegger seine Überlegungen mit einer Frage an: „Aber ist die Feier dadurch schon eine Gedenkfeier? [...] Ist die Feier durch Spiel und Gesang schon eine Gedenkfeier, eine Feier, bei der wir denken? Vermutlich kaum."3 Heidegger erscheint so offenkündig als eine Art Zugabe - der berühmte Philosophensohn der Stadt sollte bei der Feier noch ein bisschen Denken einbringen, damit es eine richtige Gedenkfeier wird. Heidegger könnte Interessantes zum Leben und Werk Kreutzers erzählen, aber das wäre nur ärmliche Unterhaltung ohne Denken. Erst wenn eine Rede aus nötigt uns auf etwas zu besinnen, kann eine Gedenkrede dafür bürgen, dass wir bei der Gedenkfeier auch denken. Denken ist ja nur im Selbstvollzug möglich - solange ich nicht denke, wird für und durch mich nicht gedacht. Besinnung ist eine formale Anzeige des Denkens. Denken heißt sich besinnen auf das, „was jeden Einzelnen von uns unmittelbar und unablässig in seinem Wesen angeht"“. Der Mensch, ist, anders gesagt, das denkende Lebewesen - aber wie wir noch sehen werden, meint Heidegger mit dieser Anzeige nicht, dass der Mensch das animal raionale oder das vernunftbegabte Lebewesen sei.

Heute im Zeitalter des vollendeten Nihilismus sind wir alle oft gedankenarm oder noch schlimmer gedankenlos. „Die Gedankenlosigkeit ist ein unheimlicher Gast, der in der heutigen Welt überall aus- und eingeht.“5 Dieser unheimlicher Gast ist ein Symptom

\footnotetext{
${ }^{2}$ Alfred Denker \& Elsbeth Büchin, Heidegger und seine Heimat, Stuttgart, Klett-Cotta, 2005, 121-132.

${ }^{3}$ Heidegger, Gelassenheit, Pfullingen, Neske, 1959, 12.

${ }^{4}$ Heidegger, Gelassenheit, 13.

${ }^{5}$ Heidegger, Gelassenheit, 13.
} 
des Nihilismus. ${ }^{6}$ Deshalb ist die Gedankenlosigkeit ein unheimlicher Gast und nicht der unheimlichste aller Gäste. Weil die Metaphysik sich im Nihilismus (in der Gestalt der planetarischen Herrschaft der Technik) vollendet hat, gibt es keine neue Denkmöglichkeiten mehr und werden wir immer gedankenloser. Heidegger kann seine Zuhörer so auf zwei Denkwege führen: der eine ist die Frage, was heißt Denken? - der andere ist die Frage nach der Technik. Begeben wir uns zuerst auf den ersten Weg.

In Sein und Zeit hatte Heidegger das implizite Seinsverständnis des Menschen phänomenologisch aufgedeckt. Ohne Denken kann es keine Menschen geben und dieses Denken ist in seiner einfachsten Form Seinsverständnis oder das In-der-Wahrheit-Sein. Der Mensch ist in seinem Dasein immer schon vom Sein in Anspruch genommen. Die Geschichte der Metaphysik ist die Geschichte der menschlichen Antwort auf diese Inanspruchnahme durch das Sein. Aber, wenn es am Ende der Geschichte der Metaphysik, d.h. im Nihilismus, mit dem Sein nichts mehr ist, verliert der Mensch sein Seinsverständnis und damit auch sein eigenes Wesen. Deshalb ist die Gefahr und die Not des heutigen Menschen so groß. „Denn man nimmt heute alles und jedes auf dem schnellsten und billigsten Weg zur Kenntnis und hat es im selben Augenblick ebenso rasch vergessen. ${ }^{67}$ Heidegger hat diesen Satz 1955 geschrieben. Heute geht es durch Rechner und Internet noch unglaublich viel schneller und billiger. In solchen Phänomenen zeigt sich die zunehmende Gedankenlosigkeit. „Der heutige Mensch ist auf der Flucht vor dem Denken."8 Aber dies bedeutet zugleich, weil das Denken die Bestimmung des Menschen ist, dass der heutige Mensch auf der Flucht vor sich selbst ist. Auch hier ist es hilfreich an den Nihilismus zu denken. Die Vollendung der Metaphysik im Nihilismus zeigt sich darin, dass der Mensch sein Seinsverständnis verliert. Dieser Verlust ist sowohl eine Folge der menschlichen Seinsvergessenheit als eine Folge der Seinsverlassenheit des heutigen Menschen. Die beiden Phänomene spiegeln einander.

Es ist leicht, um Heidegger den Vorwurf zu machen, dass es noch nie in der Geschichte so viel Forschung und Planung gegeben hat. Forschen und Planen sind doch

\footnotetext{
${ }^{6}$ Martin Heidegger, Nietzsche II, 6. erg. Auflage Stuttgart, Klett-Cotta, 1998, 43. Der Verweis auf Nietzsche ist eindeutig: „Der Nihilismus steht vor der Tür: woher kommt uns dieser unheimlichste aller Gäste?"

${ }^{7}$ Heidegger, Gelassenheit, 13.

${ }^{8}$ Heidegger, Gelassenheit, 14.
} 
Formen des Denkens und damit ist die These der Gedankenlosigkeit widerlegt. Heidegger unterscheidet zwei Formen des Denkens: das rechnende Denken und das besinnliche Nachdenken. Das Planen und Forschen ist ein eigenartiges Denken, das nie stillhält und deshalb auch nicht zur Ruhe kommt. Das rechnende Denken findet keine Inständigkeit und kann nicht warten. ${ }^{9}$ Die andere Art des Denkens nennt Heidegger das besinnliche Denken, das dem Sinn nachdenkt, der in allem waltet, was ist, d.h. dem Sinn von Sein. Wir können jetzt auch verstehen, was die große Gefahr des Nihilismus ist. Wenn nur das rechnende Denken noch übrig bleibt und das besinnliche Nachdenken verschwindet, dann geht dem Menschen sein Wesen verloren. Wenn der Mensch nur noch rechnen kann, ist die planetarische Herrschaft der Technik unüberwindbar, weil es dann keinen Raum für ein anderes Denken mehr geben kann. Bei den Griechen gab es laut Heidegger fünf Formen des In-der-Wahrheit-seins des Menschen: nous (Vernunft), sophia (Weisheit), epistèmè (Erkenntnis), phronèsis (Klugheit) und technè (Technik). Heidegger hat in seinem Werk gezeigt, wie im Laufe der Geschichte der Metaphysik, die techne oder Technik zu der einzigen Weise des In-der-Wahrheit-seins des Menschen geworden ist. Die Technik gehört zum Wesen des Menschen, da der Mensch ohne Technik überhaupt nicht leben kann. Heidegger ist nicht gegen die Technik, sondern er sieht in der Alleinherrschaft der Technik die Gefahr, dass der Mensch 80\% seine Möglichkeiten einbüßt und am Ende nicht mehr Mensch sein kann. ${ }^{10}$

Die Gefahr bei der Lektüre von Heideggers Texten ist immer, dass wir zu schnell gehen. Er hat seine Vorträge und Aufsätze sehr genau strukturiert als Denkwege und versucht uns schrittweise zu führen. Wenn wir Heidegger folgen und seine Bestimmung des rechnenden Denkens und des nachdenklichen Besinnens verstanden haben, könnten wir fragen, was der Sinn des nachdenklichen Besinnens sein könnte? Das rechnende Denken ist handfest und kann eine Unmenge von Ergebnissen vorlegen. Das besinnliche Nachdenken, d.h. das Denken, vor dem der Mensch auf der Flucht ist, schwebt doch nur über die Wirklichkeit und kann deshalb für die Durchführung des praktischen Lebens

\footnotetext{
${ }^{9}$ Heidegger, Gelassenheit, 15.

${ }^{10}$ Die Notwendigkeit des Übermenschen ist unumgehbar: entweder wird der Mensch als Arbeiter der Übermensch oder im Heideggers Sinne geht der Mensch über zu einem neuen nicht-metaphysischen Seinsverständnis und findet er ein neues Wesen.
} 
auch nichts einbringen. Und dazu kommt noch das die Besinnung für den gemeinen Menschenverstand zu hoch sei.

Für uns Heutigen sind Heideggers Analysen vermutlich verständlicher als für seine Landsleute, die seine Rede damals gehört haben. Die Naturwissenschaft ist heute noch mehr das Maß aller Dinge geworden. Nur was wir berechnen können, kann wirklich sein. An der Universität sollen die Geisteswissenschaften genauso quantifizierbar werden als die Naturwissenschaften. Jura, Medizin, Ökonomie, Architektur, Maschinebau, usw. sind die Fächer, die unmittelbaren Nutzen für das praktische Leben des Menschen haben. Wir wollen immer mehr Sicherheit haben - deshalb können wir z.B. heute alles versichern. Alles soll machbar sein - deshalb braucht unser Körper plastische Chirurgie. Da ein bisschen Kultur für unser Wohlsein nicht ganz unnütz sei, sollen auch die Künste auch noch einigermaßen gefördert werden. Wozu könnten wir überhaupt das bloße Nachdenken brauchen? Genau die Frage zeigt schon, wie technisch unser Selbstverständnis geworden ist oder, anders gesagt, wie dringend das besinnliche Nachdenken geworden ist. Das Nachdenken ist für den heutigen Mensch gerade so nützlich, weil es gar kein Nutzen hat. Das Schöne der Philosophie ist gerade, dass wir mit ihr nichts anfangen können.

Den Einwand, dass das besinnliche Nachdenken für den gemeinen Verstand zu hoch sei, kann Heidegger einfach widerlegen. Erstens fragt auch das wissenschaftliche Denken auch um Anstrengung, Studium und Übung. Für den gemeinen Verstand sind naturwissenschaftliche Theorien ebenso wenig selbstverständlich wie das bloße Nachdenken. Was Heidegger hier das besinnliche oder bloße Nachdenken nennt, ist eigentlich noch immer das phänomenologische zu den Sachen selbst gehen. Die Sache kann sich nur zeigen, wenn das Denken ihr die Freiheit lässt sich zu zeigen, so wie sie sich von sich selbst herzeigt. Dies bedeutet, dass wir nicht wie in jeder Wissenschaft einen Gegenstandsbereich festlegen sollen (Zeit, Bewegung, Raum, Natur, Leben), sondern auf den Anspruch des Seienden hören sollen. Um dies zu lernen ist eine hohe Anstrengung und eine lange Einübung notwendig. Heidegger hat 1916-1918 das Denken bei Husserl gelernt. In seinen Seminaren und Vorlesungen hat er versucht seine Studenten die Art und Weise des (philosophischen) Denkens einzuüben. Dies machte seine Lehrveranstaltungen so spannend und erfolgreich. Seine spätere Vorträge nach 1945 versuchen innerhalb ein oder zwei Stunden mit den Hörern schrittweise das Denken 
einzuüben und der Sache, die im Vortrag erörtert wird, so näher zu kommen, dass diese sich von sich selbst herzeigen kann.

Jedermann kann das Nachdenken lernen, weil der Mensch „das denkende, d.h. sinnende Wesen ist“. ${ }^{11}$ Beim Nachdenken brauchen wir nur „,beim Naheliegenden zu verweilen und uns auf das Nächstliegende zu besinnen: auf das, was, uns, jeden Einzelnen hier und jetzt, angeht".. ${ }^{2}$ Damit kann Heidegger den Übergang zum Thema seiner Gedenkrede schaffen. Was geht Heidegger und seinen Hörern jetzt und hier an? Was ist das Nächstliegende? Die einfache Tatsache, dass aus dem Boden der Heimat ein Werk der Kunst gediehen ist. Wir könnten erwarten, dass Heidegger jetzt über die Musikkunst sprechen würde; er geht aber einen anderen Weg. ,Wir werden nachdenklich und fragen: Gehört nicht zu jedem Gedeihen eines gediegenen Werkes die Verwurzelung im Boden einer Heimat?" ${ }^{13}$

Nachdenklich werden heißt sich einlassen auf das Fragwürdige und wenn wir uns auf die Fragwürdigkeit einlassen, stellt sich die Frage nach der Bodenständigkeit. Für die Weise von Heideggers Vorgehen ist es eigentümlich, dass er jetzt ein Zitat eines Dichters bringt. Die Sprache spricht noch im Werk der Dichter und wenn wir auf sie hören lernen, kommen wir die Sache, die sich in der Sprache zeigt, näher. Das Zitat lautet: „Johann Peter Hebel schreibt einmal: ,Wir sind Pflanzen, die - wir mögen's uns gerne gestehen oder nicht - mit den Wurzeln aus der Erde steigen müssen, um im Äther blühen und Früchte tragen zu können“ (Werke, ed. Altmann III, 314).“14

Heidegger erörtert diesen Satz und dieser Absatz ist für seine „Methode“ kennzeichnend. „Der Dichter will sagen: Wo ein wahrhaft freudiges und heilsames Menschenwerk gedeihen soll, muss der Mensch aus der Tiefe des heimatlichen Bodens in den Äther aufsteigen können." 15 Heidegger erörtert den Satz und sagt das, was der Dichter Johann Peter Hebel will sagen, aber nicht gesagt hat oder besser ungesagt gelassen hat. Hebel spricht nicht vom Menschenwerk, er redet von Früchten. Diese

\footnotetext{
${ }^{11}$ Heidegger, Gelassenheit, 16.

${ }^{12}$ Heidegger, Gelassenheit, 16.

${ }^{13}$ Heidegger, Gelassenheit, 16.

${ }^{14}$ Heidegger, Gelassenheit, 16.

${ }^{15}$ Heidegger, Gelassenheit, $16 f$.
} 
Früchte sind die Werke des Menschen - nicht nur Kunstwerke, sondern auch Dinge im breiten Sinne des Wortes, d.h. alles was, der Mensch baut.

Heidegger verfolgt seine Erörterung. „Äther bedeutet hier: die freie Luft des hohen Himmels, den offenen Bereich des Geistes."16 Der Äther wird von Heidegger ausgelegt einen offenen Bereich. Dieser Bereich hat er in seinem Vortrag „Bauen Wohnen Denken“"von 1951 als Geviert schon sichtbar gemacht. ${ }^{17}$ Das Geviert von Himmel und Erde und Göttlichen und Sterblichen gehört aus einer ursprünglichen Einheit zusammen. Die ersten beiden Momente des Gevierts finden wir im Hebel-Zitat: der Äther ist der Himmel und Hebel redet von der Erde. Aber er hat die innere Spannung zwischen beiden nicht gesehen. Bei Hebel sind Äther und Erde statische Phänomene ohne innere Beweglichkeit. Deshalb sollen wir noch nachdenklicher werden. ${ }^{18}$

Der Mensch wohnt zwischen Himmel und Erde als der Sterbliche, weil, wie es in Sein und Zeit heißt: „Keiner dem Anderen sein Sterben abnehmen kann. [...] Der Tod ist, sofern er ,ist', wesensmäßig je der meine. Und zwar bedeutet er eine eigentümliche Seinsmöglichkeit, darin es um das Sein des je eigenen Daseins schlechthin geht.“19 Menschsein heißt sterblich sein. Als die Sterblichen sind wir endlich. Endlichkeit kann aber nur im Gegensatz zum Unendlichen und Unsterblichen gedacht werden. Anders gesagt, als endliche Wesen haben wir immer ein Verhältnis zu dem, was über unsere Endlichkeit hinausgeht. Formal angezeigt ist dieser Bereich das, was Heidegger „die Göttlichen“ nennt.

Die Erde, in welcher wir wurzeln, gehört zur Heimat und nur die Heimat kann Bodenständigkeit spenden, aber sie muss es nicht. Die Heimat ist das Geviert und nur im Geviert kann der Mensch „dichterisch“ wohnen. ${ }^{20}$ Dichterisch wohnen bedeutet aber auch den Tod als Tod vermögen. Gerade in den Jahren nach dem Zweiten Weltkrieg haben,

\footnotetext{
${ }^{16}$ Heidegger, Gelassenheit, 17.

${ }^{17}$ Martin Heidegger, "Bauen Wohnen Denken"; in: Vorträge und Aufsätze, Pfullingen 1954, 139-156, hier $143 \mathrm{f}$.

${ }^{18}$ Heidegger, Gelassenheit, 17.

${ }^{19}$ Heidegger, Sein und Zeit, 240.

${ }^{20}$ Vgl. dazu Heidegger, “...dichterisch wohnet der Mensch..."; in: Vorträge und Aufsätze, Pfullingen, Neske, 1954, 181-198.
} 
wie Heidegger sagt, „,viele deutsche Menschen ihre Heimat verloren“. ${ }^{21}$ Der Satz ist aufschlussreich in zweierlei Hinsicht. Wenn wir erstens mit Heidegger achten auf das, was heute ist, dann sehen wir, dass immer mehr Menschen heimatlos werden. Mit der Heimat geht dem Menschen auch sein Wesen (das Wohnen im Geviert oder anders formal angedeutet: das Da seines Daseins) verloren. Es ist zweitens bemerkenswert, dass Heidegger nur von deutschen Menschen spricht. Es gab nicht nur deutsche Vertriebene sondern auch russische, polnische, französische, um von Auschwitz gar zu schweigen. Über diese Problematik werde ich am Schluss noch Einiges sagen.

In der Heimatlosigkeit zeigt sich die Bedrohung der Bodenständigkeit des heutigen Menschen. ${ }^{22}$ Bodenständigkeit ist eine formale Anzeige und keine bäuerliche Metapher. Auch wenn Heidegger zeitlebens in der Provinz geblieben ist, hat er über die Grenzen der Provinz in das Freie und Offene hinausgedacht. Bodenständigkeit gehört zum Da des Daseins. Sie ist der Boden, in dem der Mensch ständig steht. Dasein heißt sich-vor-weg-sein in einem schon-sein-bei. Über sich hinaus steht der Mensch immer schon in seinem Da. Statt Bodenständigkeit könnten wir auch Inständigkeit als formale Anzeige verwenden. Der Mensch steht aus in einem Da (Ek-sistenz und Transzendenz als Inständigkeit). Dieses Da ist die wesende Wahrheit, d.h. das Seiende im Ganzen oder das Ereignis. Dieses Ereignis ist die Tatsache, dass der Mensch vom Seyn angesprochen wird, indem er sich immer schon im Seienden im Ganzen befindet. Das Ereignis ist faktisch, weil wir es nur hinnehmen und nicht hinterfragen können. Es hat sich immer schon ereignet, wenn es Menschen oder allgemeiner, Dasein gibt. Der Verlust der Bodenständigkeit ist deshalb die größte Gefahr und die höchste Not des heutigen Menschen. Heidegger verwendet gerne Superlative, aber wir sollen uns von diesen nicht verführen lassen und nüchtern bleiben. Die größte Gefahr ist die Gefahr, die wir nicht ausweichen können und die höchste Not ist die Not, die wir nicht lindern können. Beide können wir nur gelassen hinnehmen und uns im Andenken vorbereiten auf einen neuen Zuspruch des Seyns. Warum dies so ist, werden wir verstehen lernen, wenn wir Heidegger weiter auf seinem Denkweg folgen.

Unsere Zeit ist dadurch gekennzeichnet, dass uns der Verlust der Bodenständigkeit, und damit unseres Wesens, droht. Mit Heidegger können wir dann

\footnotetext{
${ }^{21}$ Heidegger, Gelassenheit, 17.

${ }^{22}$ Heidegger, Gelassenheit, 18.
} 
fragen: „Was geschieht eigentlich in unserer Zeit? Wodurch ist sie gekennzeichnet?“.23 Heidegger übernimmt für das jetzt 1955 beginnende Zeitalter den Ausdruck ,das Atomzeitalter“, einen in der Zeit geläufiger Ausdruck. ${ }^{24}$ Aber wir können es genauso gut das Zeitalter des vollendeten Nihilismus nennen. Da das Atomzeitalter auch das Zeitalter des für das Denken auf der Flucht Sein des Menschen ist, fehlt bei allem, was zum Thema gesagt, gedacht und vor allem berichtet wird, eine Besinnung auf das Wesen des Atomzeitalters. Erst wenn wir mit Heidegger fragen, wie es möglich sei, dass die wissenschaftliche Technik die Atomenergie in der Natur entdecken und nutzbar machen konnte, können wir einen Schritt weiterkommen.

Der Anfang der Neuzeit mit der radikalen Revolution der mittelalterlichen Weltansicht, der in der metaphysischen Grundstellung von Descartes seinen Ausdruck gefunden hat, ist, wie wir schon wissen, auch der Anfang des Nihilismus. Descartes hat die Stellung des Menschen in der Welt und zur Welt völlig neu bestimmt.

Jetzt erscheint die Welt wie ein Gegenstand, auf den das rechnende Denken seine Angriffe ansetzt, denen nichts mehr soll widerstehen können. Die Natur wird zu einer einzigen riesenhaften Tankstelle, zur Energiequelle für die moderne Technik und Industrie. [...] Die in der modernen Technik verborgene Macht bestimmt das Verhältnis des Menschen zu dem, was ist. ${ }^{25}$

Heidegger arbeitet in dieser Gedenkrede auf zwei Ebenen. Auf der erste, mehr oberflächige Ebene macht er seine Hörer auf die wichtigsten Erscheinungsformen der neuen Stellung des Menschen im Ganzen des Seienden aufmerksam. Seine Beschreibungen sind höchst aktuell und vieles ist erst 50 Jahre später voll ans Licht gekommen. Das „World Wide Web“ gab es damals noch nicht in Ansätzen und macht auch deutlich wie doppeldeutig die Erfolge der Wissenschaft sind. Einerseits ist das „World Wide Web“ ein Netzwerk, das alle Menschen auf der Welt mit einander verbindet. Internet hat unseres Lebens definitiv geändert und wo es enden wird, können wir heute noch nicht wissen. Die Kommunikation ist heute unmittelbar auf der ganzen Welt. Auf unserem Rechner können wir jetzt sehen, was 10.000 Kilometer entfernt jetzt passiert. Über alles können wir unmittelbar endlos viele Informationen finden. Andererseits sind wir heute schon völlig abhängig vom Internet und können wir nicht

\footnotetext{
${ }^{23}$ Heidegger, Gelassenheit, 18.

${ }^{24}$ Heidegger. Gelassenheit, 18.

${ }^{25}$ Heidegger, Gelassenheit, $19 \mathrm{f}$.
} 
mehr ohne leben. Das hat sich innerhalb von wenigen Jahrzehnte endgültig geändert. Der Normalverbraucher ist von seinen Apparaten völlig abhängig geworden, weil er sobald es nicht mehr funktioniert rat- und machtlos ist.

Heidegger macht deutlich, dass die moderne Technik nicht nur ein Hilfsmittel für den Menschen ist, sondern dass sie auch den Menschen in Anspruch nimmt und in seinem Wesen verwandelt. Heute beginnen wir erst darauf $\mathrm{zu}$ achten, wie das Internet unsere Stellung zu und in der Welt geändert hat. Wir schreiben (SMS- und MSN-Sprache) heute anders, wir lesen anders (am Bildschirm), wir lieben anders (Sexualisierung der Gesellschaft), usw. Ob wir diese Vorgänge positiv oder negativ werten, ist nicht wichtig. Die Informationsfülle führt dazu, dass wir über unendliche Kenntnisse verfügen. Aber wie Heidegger sagt bedeutet etwas kennen noch nicht, dass wir es erkannt haben. Kennen und Erkennen sind zwei verschiedenen Formen der Wahrheitserfahrung. Informationen (Kenntnisse) sind im Internet leicht zugänglich. Das Wissen (Erkenntnisse) müssen wir uns aber erwerben. Ein großes Problem ist heute, dass wir vieles kennen, wovon wir nichts wissen.

Heidegger spricht von der in der modernen Technik verborgenen Macht. Diese verborgene Macht liegt unter den bekannten Erscheinungsformen der Technik. Aber welche Macht ist das? Diese Macht ist im Gegensatz zu allen Apparaten und Automaten, allen Anlagen und Einrichtungen nicht vom Menschen gemacht. Diese Macht hat den Menschen in seiner Macht und wie wir oben gesehen haben, ist sie der Wille zur Macht, der nur noch sich selbst will. Die Vollendung der Metaphysik in dem Nihilismus zeigt sich auch darin, dass das Leben in die Hand des Biochemikers, „der die lebendige Substanz nach Belieben ab- und aufbaut und verändert“, gelegt ist. ${ }^{26}$ Heidegger war 1955 schon hellsichtig - heute können wir viele von den Problemen, die Heidegger angedeutet hat, in einem ganz anderen Umfang erfahren. Die Gen- und Biotechnologie führt zu großen ethischen Fragen. Der Mensch ist heute zumindest zum Teil machbar geworden, d.h. der Mensch betrachtet sich selbst als Gegenstand und als Gegenstand ist er der Technik ausgeliefert. Wir versuchen heute die Krankheit technisch zu verbannen. Es wäre zu leicht und einfach zu behaupten, dass wir heute nur noch technisch die Krankheit bestreiten, ohne die Gesundheit zu fördern. Es ist aber nicht zu verneinen, dass wir

${ }^{26}$ Heidegger, Gelassenheit, 22. 
Krankheit kaum noch als zum Wesen des Menschen und des Lebens gehörig verstehen. Menschsein heißt dagegen eigentlich nicht nur gesund, sondern auch krank sein können.

Dass durch die moderne Technik eine ungeheure Veränderung der Welt stattgefunden hat und noch immer stattfindet, ist offenkündig und unumkehrbar. Heidegger sieht als Phänomenologie noch etwas anderes und entscheidenderes: „Dabei ist jedoch das eigentlich Unheimliche nicht dies, daß die Welt zu einer durch und durch technischen wird. Weit unheimlicher bleibt, daß der Mensch für diese Weltveränderung nicht vorbereitet ist, daß wir es noch nicht vermögen, besinnlich denkend in eine sachgemäße Auseinandersetzung mit dem zu gelangen, was in diesem Zeitalter eigentlich heraufkommt. “27

Auch in seiner Gedenkrede ist Heidegger davon überzeugt, dass heute der Übermensch gefordert sei. Das war schon Thema in seiner Rektoratsrede von 1933 und in seiner damaligen Jünger-Deutung. Auch in „Wer ist Nietzsches Zarathustra?“ stellt er die Frage nach dem Übermenschen. Der entscheidende Unterschied ist, dass Heidegger 1933/34 denkt, dass der Übermensch machbar wäre; 1955 weiß er besser. Der Wille zur Macht verbirgt sich im Wesen der Technik, deshalb ist jeder Versuch die Technik Herr zu werden noch immer eine Steigerung des Willens zur Macht. Deshalb soll der Übermensch nichts tun, sondern lernen das Seiende sein zu lassen. Der Übermensch ist der Mensch, der über die Brücke des besinnlichen Nachdenkens geht. Es ist diese Möglichkeit eines anderen Denkens, die uns erlauben kann ein anderes Verhältnis zur Technik zu finden. Heidegger hat immer wieder darauf bestanden, dass das Wesen der Physik nichts Physikalisches, dass das Wesen der Technik nichts Technisches und dass das Wesen des Menschen nichts Menschliches sei. Damit meint er das die Physik die Frage, „was die Physik sei?“”, nicht mit ihren Methoden beantworten kann - es handelt sich ja nicht um ein physisches Problem. Aber auch das Wesen der Technik ist nichts Technisches und dies bedeutet, dass das Problem der unaufhaltsamen Übermacht der Technik nicht technisch gelöst werden kann. Wenn wir versuchen die fossilen Brennstoffen durch umweltfreundlichere Energiequellen $\mathrm{zu}$ ersetzen, ändern wir an unserem Verhältnis zur Natur (und zur Welt im Ganzen) noch immer nichts.

Heidegger sieht jetzt als seine denkerische Aufgabe, dass er bei jeder Gelegenheit versuchen soll das besinnliche Denken wach zu rufen. Er versucht es auch mit seiner

${ }^{27}$ Heidegger, Gelassenheit, 22. 
Gedenkrede in Meßkirch. Statt Antworten zu geben und als Führer tätig zu werden, stellt er jetzt Fragen: „Könnte nicht, wenn schon die alte Bodenständigkeit verloren geht, ein neuer Grund und Boden dem zurückgeschenkt werden, ein Boden und Grund, aus dem das Menschenwesen und all sein Werk auf eine neue Weise und sogar innerhalb des Atomzeitalters zu gedeihen vermag? Welches wäre der Grund und Boden für eine neue Bodenständigkeit?" 28

Heidegger spricht hier vom Menschenwesen und nicht vom Menschen. Dies ist das Da des Daseins, d.h. die Lichtung innerhalb welcher der Mensch aus-steht oder existiert. Dieses Da ist der Grund und Boden für eine neue Bodenständigkeit. Dieses Da gibt es aber nur, wenn das besinnliche Denken wach wird. Deshalb liegt das, wonach Heidegger fragt, sehr nah. Selbst so nah - es ist das Wesen des Menschen -, dass wir es allzu leicht übersehen. Der Weg zu diesem Nah ist die Brücke, über welche wir in das besinnliche Denken geraten können. Wir sollen nicht versuchen die Gegenständlichkeit der objektiven Welt zu ändern, sondern auf unserem innersten und eigentlichsten Wesen achten: das Denken. ${ }^{29}$ Die Besinnung befreit uns aus dem vorstellenden Denken und damit aus der Wirklichkeit der technischen Welt. Die Besinnung ist der Schritt zurück in den Boden der Technik. Wir können mit Heidegger die Probe machen. Wir können heute nicht mehr ohne die Einrichtungen, Apparate und Maschinen der technischen Welt leben. Es ist darum auch sinnlos, „die technische Welt als Teufelswerk zu verdammen“. ${ }^{30}$ Dennoch sollen wir nicht in die Knechtschaft zur Technik geraten. Heideggers Lösung ist ein gleichzeitiges Ja und Nein sagen zu den technischen Gegenständen, d.h., dass wir einerseits die technischen Gegenstände benutzen (wir können ja nicht ohne sie leben) und sie so im Gebrauch nehmen, wie sie genommen werden müssen. Andererseits sollen wir versuchen die technischen Gegenstände auf sich beruhen zu lassen, weil sie uns das Leben ermöglichen und erleichtern sollen, ohne uns im Innersten und Eigentlichen anzugehen. Die große Gefahr ist, dass wir uns selbst nur noch technisch als Gegenstand betrachten und vergessen, dass die Technik nur einer Weise des In-der-Wahrheit-Seins des

\footnotetext{
${ }^{28}$ Heidegger, Gelassenheit, 23.

${ }^{29}$ Heidegger, Gelassenheit, 24.

${ }^{30}$ Heidegger, Gelassenheit, 24.
} 
Menschen ist. Heidegger nennt die Haltung des gleichzeitigen Ja und Nein sagen zu den technischen Gegenständen mit einem alten Wort „die Gelassenheit zu den Dingen““.31

In dieser Haltung lassen wir die Dinge nicht länger nur Gegenstände sein. Wir entbergen das Seiende, anders gesagt dann nicht nur als Gegenstand, das gegenüber uns als Subjekt steht, sondern lassen es auch als Ding, das Erde und Himmel und Sterblichen und Göttlichen sammelt, sein. Aber welcher Sinn waltet im sich wandelnden menschlichen Verhältnis zur Natur und Welt? Damit meint Heidegger den Umschlag der neuzeitlichen Metaphysik in den Nihilismus und die planetarische Herrschaft der modernen Technik. Wir wissen nicht, wo dieser Wandel enden wird. „Der Sinn der technischen Welt verbirgt sich. “32 Dennoch werden wir überall in der technischen Welt von einem Sinn angerührt, der, wenn wir darauf achten, uns angeht. Diese Haltung der Achtsamkeit und der Besinnung nennt Heidegger „,die Offenheit für das Geheimnis“. ${ }^{33}$ Die Gelassenheit zu den Dingen und die Offenheit für das Geheimnis gehören zusammen und gewähren uns die Möglichkeit eines nicht nur technischen Aufenthaltes in der Welt. Sie gehen uns damit Aussicht auf eine neue Bodenständigkeit und eröffnen den Bereich einer neuen Heimat.

Die größte Gefahr im Atomzeitalter ist nach Heidegger nicht die Gefahr eines Dritten Welt- und Atomkriegs, sondern daß ,das rechnende Denken als das einzige in Geltung und Übung bliebe“ “. ${ }^{34}$ Warum ist das die größte Gefahr und die höchste Not? Weil dies zur totalen Gedankenlosigkeit führen würde. Und dann? „Dann hätte der Mensch sein Eigenstes, daß er nämlich ein nachdenkendes Wesen ist, verleugnet und weggeworfen? Darum gilt es das Wesen des Menschen zu retten. Darum gilt es, das Nachdenken wach zu halten.“35 Wenn der Mensch sein Eigenstes verleugnet, kann es keine Dinge mehr geben und wird die Erde endgültig und unumkehrbar verwüstet.

Die Gelassenheit zu den Dingen und die Offenheit für das Geheimnis gedeihen nur aus dem, was Heidegger ,einem unablässigen herzhaften Denken“ nennt. ${ }^{36}$ Das

\footnotetext{
${ }^{31}$ Heidegger, Gelassenheit, 25.

${ }^{32}$ Heidegger, Gelassenheit, 26.

${ }^{33}$ Heidegger, Gelassenheit, 26.

${ }^{34}$ Heidegger, Gelassenheit, 27.

${ }^{35}$ Heidegger, Gelassenheit, 27.

${ }^{36}$ Heidegger, Gelassenheit, 27.
} 
Denken soll nicht rational, sondern herzhaft sein, weil es uns Eigenstes ist. Wenn die Gelassenheit und die Offenheit in uns erwachen, dürften wir auf einen Weg gelangen, der zu einer neuen Heimat führt und eine neue Bodenständigkeit ermöglicht. ${ }^{37}$ Es gibt in Heideggers Gedenkrede ein Problem, was wir leicht übersehen können. Er macht absichtlich den gleichen Fehler wie Jünger. Heidegger spricht in seiner Rede eine Sprache, aber sobald er vom besinnlichen Denken redet, befindet er sich in einem radikal anderen Sprachbereich und sollte er eigentlich eine ganz andere Sprache verwenden. Die Sprache der Besinnung soll eine nicht-metaphysische Sprache sein. Proben von dieser anderen Sprache finden wir in seiner Erläuterung zu Dichtern wie Hölderlin, Rilke, George und Trakl, in seinen Erörterungen von den frühgriechischen Denken und Dichtern, und in seinen sogenannten Winken, sowie wir sie z.B. in Aus der Erfahrung des Denkens finden.

Zum Abschluss möchte ich die wichtigsten Ergebnisse zusammenfassen und noch kurz auf die Folgen von Heideggers Denkweise eingehen. Wir sollen nie vergessen, dass Heidegger zeitlebens seiner phänomenologischen Methode treu geblieben ist. Der Ausgangspunkt seines Denkens ist die Faktizität des Daseins. Da das Dasein jemeinig ist, kann das Denken sich nur auf das jemeinige Heute beziehen, d.h. jedes ernsthafte Denken fängt mit einer Analyse der eigenen Zeit an. Heidegger deutet das 20. Jahrhundert als die Vollendung der Metaphysik. Diese Vollendung zeigt sich sowohl in der planetarischen Herrschaft der Technik als im Nihilismus. Der Nihilismus ist die letzte Möglichkeit der Metaphysik und erklärt, warum es heute mit dem Sein nichts mehr ist und warum es seit Hegel keine neue Metaphysik mehr gegeben hat und geben kann. Die Geschichte der Metaphysik ist, wie Heidegger in seiner großartigen Interpretation der Geschichte der Philosophie gezeigt hat, das Abbild der Geschichte des Seyns. Die Frage nach dem Übermenschen ist die Frage nach der Möglichkeit eines anderen, d.h. nichtmetaphysischen Denkens.

Heidegger interpretiert die Geschichte der Metaphysik als die Geschichte des Nihilismus. Der Nationalsozialismus erscheint dann als schlimmste Vollendung des Nihilismus. In seinen Bremer Vorträgen, die er 1949 unter dem Gesamttitel „Einblick in das was ist" gehalten hat, gibt es zwei Passagen, die oft kritisiert werden.

\footnotetext{
${ }^{37}$ Heidegger, Gelassenheit, 28.
} 


\begin{abstract}
„Das bäuerliche Tun fordert den Ackerboden nicht heraus; es gibt vielmehr die Saat den Wachstumskräften anheim; es hütet sie in ihr Gedeihen. Inzwischen ist jedoch auch die Feldbestellung in das gleiche Be-stellen übergegangen, dass die Luft auf Stickstoff, den Boden auf Kohle und Erze stellt, das Erz auf Uran, das Uran auf Atomenergie, diese auf bestellbare Zerstörung. Ackerbau ist jetzt motorisierte Ernährungsindustrie, im Wesen das Selbe wie die Fabrikation von Leichen in Gaskammern und Vernichtungslagern, das Selbe wie die Blockade und Aushungerung von Ländern, das Selbe wie die Fabrikation von Wasserstoffbomben." ${ }^{\text {"38 }}$
\end{abstract}

Dieses Zitat ist beim ersten Lesen provozierend oder sogar schockierend. Ackerbau ist heute im Wesen das Selbe wie die Fabrikation von Leichen in Gaskammern und Vernichtungslagern, wie könnte das wahr sein. Augenscheinlich stellt Heidegger hier Ackerbau an Auschwitz gleich. Dennoch sagt Heidegger dies nicht. Der moderne Ackerbau ist Bioindustrie geworden und ein Austrag des Nihilismus. In dieser Industrie werden Lebewesen vergegenständlicht und nur noch gesehen als Industrieprodukt. Mit dem Tier ist es nichts mehr; es ist belang- und wertlos geworden. Die Produktion von Leichen ist für Heidegger das Selbe. Verharmlost er auf dieser Weise Auschwitz nicht? Verliert die Shoah so nicht ihre Einmaligkeit und unverständliche Schrecklichkeit? Dase Selbe ist aber sicherlich nicht das Gleiche. Einerseits ist die Produktion das Selbe, weil auch sie ein Austrag des Nihilismus ist. Andererseits aber ist sie auch entscheidend etwas Anderes, da in Auschwitz Menschen nur noch als Vernichtungsgegenstand gesehen wurden und als gesichtsloses Material vernichtet wurde. Die Shoah ist, wenn wir Heidegger folgen, der grauenhafte Austrag des Nihilismus. Damit wird sie auch geschichtlich eingeordnet. Der Nihilismus ist die Möglichkeitsbedingung von Auschwitz. Heidegger wurde vermutlich mir zustimmen, wenn ich behaupte, dass die Materialschlachten des Ersten Weltkrieges eine Vorstufe von Auschwitz sind. Die Shoah ist (hoffentlich) einmalig und unvergleichbar mit allen anderen historischen Ereignissen, aber sie ist auch Teil der menschlichen Geschichte. Es gibt in Heideggers dritten Vortrag „Die Gefahr“ einige ganz wichtigen Absätze.

„Hunderttausende sterben in Massen. Sterben sie? Sie kommen um. Sie werden umgelegt. Sterben sie? Sie werden Bestandstücke eines Bestandes der Fabrikation von Leichen. Sterben sie? Sie werden in Vernichtungslagern unauffällig liquidiert. Und auch ohne Solches - Millionen verelenden jetzt in

\footnotetext{
${ }^{38}$ Martin Heidegger, "Das Ge-Stell”; in: M. Heidegger, Bremer und Freiburger Vorträge. 1. Einblick in das was ist. Bremer Vorträge 1949. 2. Grundsätze des Denkens. Freiburger Vorträge 1957, hrsg. von Petra Jaeger (GA 79), Frankfurt am Main 1994, 27.
} 
| Dossiê | Martin Heideggers Festrede Gelassenheit: die Frage nach dem Wesen der Technik und dem Denken des Menschen | Dr. Alfred Denker |

China durch den Hunger in ein Verenden. Sterben aber heißt, den Tod in sein Wesen austragen. Sterben können heißt, diesen Austrag vermögen. Wir vermögen es nur, wenn unser Wesen das Wesen des Todes mag.“39

Wenn Sterblichkeit zum Wesen des Menschen gehört, dann wurde in Auschwitz den Opfern ihre Sterblichkeit geraubt und damit auch ihre Menschlichkeit. Heidegger hat durch seine Phänomenologie hier etwas Wichtiges getroffen. Die Opfern wurden nicht „nur“ getötet, sondern in ihrem Wesen vernichtet. Gerade diese Vernichtung der Menschlichkeit ist das Unwesen der Shoah.

Recebido em: 30/07/2020 | Aprovado em: 01/08/2020

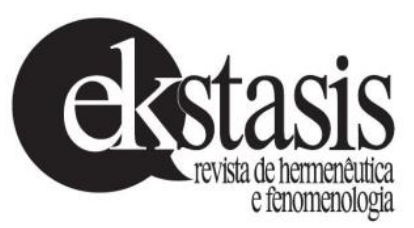

${ }^{39}$ Martin Heidegger, "Die Gefahr”, in: GA 79, 56. 